\title{
Plasma Homocysteine and Vascular Disease
}

\section{Kelly_Marie Elian ${ }^{*}$}

\author{
* To whom correspondence should be addressed: School of Dietetics and Nutrition, McGill University, \\ MacDonald-Stewart Building, 21111 Lakeshore Road, St. Anne de Bellevue, QC, Canada \\ H9X 3V9
}

\section{INTRODUCTION}

Vascular disease is the leading cause of death and disability in the Western World: atherosclerosis is responsible for the majority of deaths in virtually all westernized societies; pulmonary embolism, a complication of deep venous thrombosis, is the leading cause of morbidity and mortality, resulting in approximately 50,000 deaths per year in the United States; approximately 1.5 million acute myocardial infarcts occur each year, each infarction bearing a 30\% mortality rate; and strokes represent the third leading cause of death in developed countries (1).

While the adverse effects of hypertension, hypercholesterolemia, diabetes, and smoking on the vascular system have received widespread publicity, another important risk factor for vascular disease--less wellknown outside the medical community but recently gaining increasing attention in the literature is an elevated plasma level of the amino acid homocysteine. Bolstered by findings of an association between

hyperhomocysteinemia and a variety of vascular disorders, theories implicating homocysteine in the pathogenesis of these disorders have inspired researchers to explore novel approaches in the prevention and treatment of vascular disease. With the biochemistry of homocysteine metabolism already well understood, vitamin supplementation has emerged as a promising strategy for reduction of plasma homocysteine levels, with consequent arrest or reversal of the postulated pathogenic mechanisms involved in homocysteineassociated disorders of the vasculature, and, thus, potential prevention and treatment of vascular disease in hyperhomocysteinemic patients.

\section{HOMOCYSTEINE METABOLISM PATHWAYS}

Homocysteine is a sulfur-containing amino acid that, rather than functioning as one of the 20 amino acid building blocks of all proteins, serves as a metabolic intermediary formed by the demethylation of dietary methionine. In plasma, homocysteine exists in three forms: i.) homocysteine, ii.) the disulfide homocysteine, and iii.) the mixed disulfide homocysteine cysteine. There is almost no free homocysteine in normal plasma, as nearly all homocysteine, irrespective of form, is bound to plasma proteins (2).

The transsulfuration pathway is the series of reactions by which the four-carbon amino acid methionine is converted into the three-carbon amino acid cysteine (Fig. 1). Methionine, a dietary amino acid, is first converted to homocysteine in a series of reactions involving the $S$-adenosylmethionine (SAM) intermediate. Cystathionine- $\beta$-synthase and its cofactor pyridoxal 5' phosphate then act to combine homocysteine with 
serine, resulting in the formation of cystathionine, which is cleaved by cystathionine-gamma-lyase to form alpha-ketobutyrate and cysteine. The critical step, irreversible in humans, is the formation of cystathionine from homocysteine. Thus, a deficiency of cystathionine- 3 -synthase results in the abnormal accumulation of homocysteine. Homocysteine can be remethylated to reform methionine by means of reactions requiring homocysteine methyltransferase (which requires $\mathrm{B}_{12}$ as a cofactor), methylene-tetrahydrofolate reductase, and the methyl-group donor $\mathrm{N}^{5}$-methyl-tetrahydrofolate (THF) or betaine (an oxidized metabolite of choline). A deficiency in any of these enzymes, cofactors, or substrates can also result in hyperhomocysteinemia.

Homocystinuria is a rare genetic abnormality caused most frequently by a homozygous deficiency of the enzyme cystathionine- $\beta$-synthase $(3,4)$, but can also be due to a deficiency of methyltransferase $(5)$ or methylenetetrahydrofolate reductase (6). It is characterized by a dramatic increase of homocysteine in plasma, tissue, and urine $(7,8)$. Patients with this disorder exhibit multiple abnormalities, including dislocated lens, mental retardation, skeletal deformities, and severe vascular disease (9). The latter has been found to consist of widespread arterial and venous thrombosis as well as marked arterial intimal proliferation and plaque formation (10). These clinical findings led researchers to investigate the possible role of defective homocysteine metabolism in the genesis and progression of vascular disease.

\section{THEORIES OF PATHOGENESIS}

In 1969, McCully suggested that homocysteine has a direct toxic effect on cultured endothelial cells (5), and that, in experimental models of atherogenesis, an elevated plasma homocysteine concentration acts in concert with dietary lipids (11) to produce intimal lesions. He postulated that excess homocysteine results in the abnormal sulfation of proteoglycans through the intermediary substance homocysteine thiolactone, a known cellular toxin $(12,13)$. Recent studies suggest that the vascular endothelium in patients with cystathionine- $3-$ synthase deficiency has heightened susceptibility to homocysteine-induced injury, as homocysteine has been shown to increase DNA synthesis in vascular smooth muscle cells in a manner consistent with early atherosclerotic lesions. Furthermore, it has been demonstrated to induce these cells to proliferate, while impeding the regeneration of damaged endothelial cells $(14,15)$. Homocysteine has also been shown to cause oxidation of low-density lipoproteins (LDLs), which are cytotoxic and have been implicated in endothelial injury (16-18). Effects of homocysteine on vascular hemostatic properties, including inhibition of protein C activation and decreased cell surface expression of thrombomodulin have also been demonstrated, suggesting that homocysteine may contribute to development of thrombosis (19).

\section{ASSOCIATIONS WITH VASCULAR DISEASE}

In a study of the association between cardiovascular risk factors and total plasma homocysteine, Nygard et al. found that the level of total plasma homocysteine increases with age, is higher in men than in women, and increases markedly with the number of cigarettes smoked daily in all age groups, particularly in women (20). When the effects of age, sex, and smoking were combined, older male smokers were discovered to have plasma homocysteine levels $4.8 \mathrm{mmol} / \mathrm{L}$ higher than young non-smoking women. Furthermore, total plasma homocysteine correlated directly with total cholesterol, blood pressure, and heart rate, and was inversely related to physical activity, even after multivariate adjustment. Selhub et al., similarly observed that homocysteine concentration increases significantly with age (21): in a cohort study, the occurrence of high homocysteine concentration (defined as $>14 \mathrm{mmol} / \mathrm{L}$ ) was over $40 \%$ for those aged 80 years and older, as compared to $29 \%$ for the general population.

Various investigations have revealed an association between hyperhomocysteinemia and atherosclerotic disease. In one study, $72 \%$ of patients with hyperhomocysteinemia had sustained an atherosclerotic vascular event, a much higher percentage than the $44 \%$ of patients with normal homocysteine (22). After covariance adjustment for age, sex, race, and Quetelet body mass index, subjects who had experienced atherosclerotic events had higher mean homocysteine and cystathionine levels than those who had not experienced an event. 
Selhub et al. likewise found an association between the risk of developing carotid artery atherosclerosis and plasma homocysteine concentration (23). After stratification of plasma homocysteine concentrations into quartiles, the prevalence of arterial stenosis was shown to be $27 \%$ in the lowest quartile and $58 \%$ in the highest quartile for men, and a range of 31-39\% across quartiles for women.

Studies of the association between hyper-homocysteinemia and acute vascular events, such as stroke and acute myocardial infarction, are also underway. In a nested case-control-study involving one hundred and forty-one cases of stroke identified during follow-up of men with no history of stroke at screening, total plasma homocysteine concentrations were significantly higher in these cases as compared with matched controls (mean $13.7 \mathrm{mmol} / \mathrm{L}$ versus $11.9 \mathrm{mmol} / \mathrm{L}$ ) (26). This relationship was not weakened after adjustments for other risk factors were made, suggesting that total plasma homocysteine level is a strong independent risk factor for stroke. However, the significance of the finding that patients recovering from acute myocardial infarction had significantly higher plasma values of both homocysteine and cysteine at six weeks follow-up than during the acute phase (24-36h after the onset of symptoms) has yet to be determined (25).

In addition to the aforementioned disorders involving the arterial circulation, hyperhomocysteinemia has been implicated in venous thrombosis. When defined as a homocysteine concentration above the 90th percentile found in controls during fasting or after methionine loading, $25 \%$ of patients with recurrent venous thrombosis had hyperhomocysteinemia as compared to $10 \%$ in age-matched controls (24). The findings also demonstrated that the risk of venous thrombosis increases with elevated homocysteine concentration, both fasting and non-fasting.

\section{THE ROLE OF VITAMINS}

Armed with the knowledge of homocysteine biochemistry, many researchers have sought to study the effects of a variety of nutritional components--including folate, pyridoxine, vitamin $\mathrm{B}_{12}$, and betaine, both separately and in combination--on plasma homocysteine concentration. A better understanding of the nutritional determinants of plasma homocysteine has led researchers to experiment with vitamin supplementation, both in normal subjects and in those with vitamin deficiencies.

\section{Nutritional Determinants of Plasma Homocysteine}

Several studies suggest a strong association between intake and serum levels of certain vitamins and plasma homocysteine concentration $(21,30-32)$. Several authors have found that serum or red blood cell folate levels, as well as serum levels of vitamin $\mathrm{B}_{12}$, even within their normal ranges, are strong determinants of plasma homocysteine concentration (30-32). In one study, subjects in the lowest $20 \%$ for plasma folate level and in the lowest $30 \%$ for folate intake were found to have mean plasma homocysteine concentrations significantly greater than for those in the top $10 \%$ (21). Similarly, mean homocysteine concentrations were significantly elevated for subjects in the lowest $10 \%$ for serum vitamin $B_{12}$ relative to the highest $10 \%$. However, vitamin $\mathrm{B}_{12}$ intake appeared unrelated to mean homocysteine concentration. In addition, mean homocysteine concentrations were significantly elevated among subjects in the lowest $10 \%$ for plasma pyridoxal $5^{\prime}$ phosphate relative to those in the top $10 \%$. For subjects in the lowest $30 \%$ for vitamin $\mathrm{B}_{6}$ intake, mean homocysteine concentrations were significantly elevated. Considering both plasma values and intake of all three vitamins combined, the prevalence of high homocysteine was almost six-fold greater among subjects in the lowest $30 \%$ when compared with subjects in the top $70 \%$.

\section{Vitamin Supplementation}

Naureth et al. investigated the effect of eight intramuscular vitamin injections, given over 20 days, containing $1 \mathrm{mg}$ vitamin $\mathrm{B}_{12}, 1.1 \mathrm{mg}$ folate, and $5 \mathrm{mg}$ vitamin $\mathrm{B}_{6}$, on serum homocysteine concentration in an elderly 
population (27). Reduction of elevated homocysteine concentrations to levels within the normal range occurred with significantly increased frequency among those receiving vitamins as compared to the placebo group, with the difference in concentrations being greatest on day 21 . Of the subjects with increased homocysteine and normal pre-treatment serum values of all three vitamins, homocysteine normalized in $84 \%$ after vitamin supplementation compared with only $20 \%$ of placebo recipients. In another study, a mean of 15 weeks of therapy with folic acid $(5 \mathrm{mg} /$ day $)$ and pyridoxine $(100 \mathrm{mg} /$ day) in 10 hyperhomocysteinemic patients resulted in normalization of median homocysteine level in 7 of the patients (22).

The homocysteine-lowering effect of daily combined treatment with $250 \mathrm{mg}$ vitamin $\mathrm{B}_{6}$ and $5 \mathrm{mg}$ folic acid was explored by van den Berg et al. in mildly hyperhomocysteinemic patients with vascular disease (28). After six weeks of treatment, normalization of plasma homocysteine concentration after methionine loading occurred in $92 \%$ of patients. Fasting homocysteine levels normalized in $91 \%$ of a subset of patients who exhibited hyperhomocysteinemia when fasting. Normalization of the post-load plasma homocysteine concentration occurred in the remaining $9 \%$ after another six weeks of therapy with the addition of $6 \mathrm{~g} / \mathrm{day}$ betaine anhydrous. Although pre-treatment levels of vitamin $\mathrm{B}_{6}$, vitamin $\mathrm{B}_{12}$, and folic acid were normal, treatment with these supplements resulted in mean reductions of post-load and fasting plasma homocysteine concentrations of $48 \%$ and $51 \%$, respectively, independent of the location of vascular disease.

Betaine, together with pyridoxine and folate, have been found to produce sustained lowering of plasma homocysteine in patients with homocysteinuria non-responsive to pyridoxine alone (29). The critical underlying mechanism is presumably an increased flux through the enzyme betaine homocysteine methyl transferase, which mediates the remethylation of homocysteine to methionine. Reductions in serum homocysteine were observed after 3-4 weeks of therapy with $6 \mathrm{~g} /$ day betaine in addition to conventional pyridoxine, folate, and/or vitamin $\mathrm{B}_{12}$ therapy.

Studies suggest that homocysteine levels can be reduced by long-term daily intake of multivitamins containing $0.4 \mathrm{mg}$ folic acid $(25,31,34)$. In one study, patients regularly taking multivitamins including only $0.2-0.4 \mathrm{mg}$ folic acid have been shown to have significantly lower (22\%) plasma homocysteine levels than those not taking multivitamins (31). Patients with acute MI who received either $2.5 \mathrm{mg}$ or $10 \mathrm{mg}$ folic acid daily for six weeks demonstrated significant reductions (27\% in both groups) in their plasma homocysteine levels, despite normal pre-treatment folate and cobalamin concentrations (25). With a few exceptions, the response to folic acid was proportional to the pre-treatment homocysteine values.

The existence of a homocysteine-lowering effect of folic acid in subjects without a folate deficiency may be explained by the fact that excess folic acid, after conversion to methyltetrahydrofolate, increases the rate at which homocysteine is remethylated to methionine. Vitamin $\mathrm{B}_{12}$ and pyridoxine, on the other hand, do not decrease plasma homocysteine in the absence of an underlying deficiency, since they are coenzymes rather than cosubstrates (35). At the same time, hyperhomocysteinemia caused by a deficiency of vitamin $\mathrm{B}_{12}$ does not respond to therapy with folic acid alone (36).

The potential beneficial effects of vitamin supplementation on the risk of developing vascular disease are supported by findings that increased vitamin intake decreases plasma homocysteine levels and by studies showing an association between folate levels and vascular disease. Morrison found a $69 \%$ greater risk of coronary mortality among patients in the lowest $25 \%$ of serum folate compared with the highest $25 \%$ (33). Similarly, Selhub demonstrated that the prevalence of carotid artery stenosis with $>25 \%$ occlusion, which is correlated with increased risk of stroke and CAD, was inversely related to both folate concentration and pyridoxal 5' phosphate concentration after adjusting for age, sex, and other risk factors (23).

Food fortification with folic acid could have a considerable effect on the prevention of vascular disease, in addition to decreasing the risk of neural tube defects (37). On the other hand, high doses of folic acid may 
mask the hematologic manifestation of unrecognized pernicious anemia ( $\mathrm{B}_{12}$ deficiency), and thereby permit its neurologic manifestations, which may be severe and include spinal cord damage, to progress (38). Oakley advocates folic acid fortification of a food staple, maintaining that potential harm secondary to overconsumption would primarily be relevant only to those taking folate supplements (37). An oral dose of vitamin $B_{12}$ or the addition of $0.4 \mathrm{mg}$ vitamin $B_{12}$ to the folate supplement would correct any possible deficiency in this situation, and decrease the associated cardiovascular risks.

\section{FUTURE DIRECTIONS}

The literature reviewed here provides considerable evidence that hyperhomocysteinemia is strongly and independently related to vascular disease, including atherosclerosis, carotid artery stenosis, venous thrombosis, myocardial infarction, and stroke. High concentrations of homocysteine are frequently observed in patients with coronary, cerebrovascular, or peripheral arterial disease, as well as in subjects with subclinical atherosclerosis. Further prospective studies are required to establish causality between increased plasma homocysteine concentration and vascular disease and to approach a better understanding of pathogenesis.

Both nutrient intake and serum levels affect plasma homocysteine concentration. Supplementation with folic acid, vitamin $\mathrm{B}_{6}$, vitamin $\mathrm{B}_{12}$, and/or betaine is effective in rapidly normalizing plasma homocysteine concentration. To investigate the effect of vitamin therapy on the progress and prevention of vascular disease in patients with hyperhomocysteinemia, research involving long-term intervention is needed. Moreover, studies examining a dose-response relationship are essential, as the optimal level of vitamin supplementation has yet to be determined. Continued research in this field promises to facilitate a better understanding of the risks and benefits of vitamin supplementation, and may lead to future fortification of foods in order to reduce the burden of vascular disease.

\section{REFERENCES}

1. Isselbacher K, Braunwald E, Wilson JD, eds. Harrison's Principles of Internal Medicine. New York: McGraw-Hill, 1994; 1066-1077, 1106, 1140-1141, 1214-1215, 2233-2251.

2. Kang SS, Wong PWK, Cook HY, et al. Protein bound homocysteine: a possible risk factor for coronary artery disease. Journal of Clinical Investigation 77: 1482-6; 1986.

3. Mudd SH, Finkelstein JD, Irrevere F, et al. Homocystinuria: an enzymatic defect. Science 143: 1443-5; 1964.

4. Hu FL, Gu Z, Kozich V, et al. Molecular basis of cystathionine beta synthase deficiency in pyridoxine responsive and nonresponsive homocystinuria. Human Molecular Genetics 2:1857-60; 1993.

5. McCully KS. Vascular pathology of homocysteinemia: implications for the pathogenesis of arteriosclerosis. American Journal of Pathology 56: 111-28; 1969.

6. Mudd SH, Uhlendorf BW, Freeman JM, et al. Homocystinuria associated with decreased methylenetetrahydrofolate reductase activity. Biochemical and Biophysical Research Communications 46: 905-12; 1972.

7. Carson NAJ, Neill DWS. Metabolic abnormalities detected in a survey of mentally backward individuals in Northern Ireland. Archives of Disease in Childhood 37: 505-13; 1962.

8. Gerritsent T, Vaughn JG, Waisman HA. The identification of homocysteine in the urine. Biochemical and 
Biophysical Research Communications 9: 493-6; 1962.

9. Mudd SH, Levy HL. Disorders in transsulfuration. In: Scriver CR, Beaudet AL, Sly WS, Valle D, eds. The metabolic basis of inherited disease, 6th ed. New York: McGraw Hill, 1989.

10. Mudd SH, Skovby F, Levy HL, et al. The natural history of homocystinuria due to cystathionine beta synthase deficiency. American Journal of Human Genetics 37: 1-31; 1985.

11. McCully KS, Olszewski AJ, Vezeridis MP. Homocysteine and lipid metabolism in atherogenesis: effect of the homocysteine thiolactonyl derivatives, thioretinaco and thioretinamide. Atherosclerosis 83: 197-206; 1990.

12. McCully KS. Homocysteine theory of arteriosclerosis: development and current status. In: Gotto Am Jr, Paoletti R, editors. Atherosclerosis reviews. New York: Raven P, 1983.

13. Dudman NPB, Hicks C, Lynch JF, et al. Homocysteine, thiolactone disposal by human arterial endothelial cells and serum in vitro. Arteriosclerosis and Thrombosis 11: 663-70; 1991.

14. Wang J, Dudman NPB, Wilcken DEL, et al. Homocysteine catabolism: levels of 3 enzymes in cultured human vascular endothelium and their relevance to vascular disease. Atherosclerosis 97: 97-106; 1992.

15. Tsai J, Perrella MA, Yoshizumi M, et al. Promotion of vascular smooth muscle cell growth by homocysteine: a link to atherosclerosis. Proceedings of the National Academy of Sciences of the United States of America 91: 6369-73; 1994.

16. Heinecke JW, Rosen H, Chait A. Iron and copper promote modification of low density lipoprotein by human arterial smooth muscle cells in culture. Journal of Clinical Investigation 74:1890-4; 1984.

17. Heinecke JW, Rosen H, Suzuki LA, et al. The role of sulfur containing amino acids in superoxide production and modification of low density lipoproteins by arterial smooth muscle cells. Journal of Biological Chemistry 262: 10098-103; 1987.

18. Parthasarathy S. Oxidation of low density lipoprotein by thiol compounds lead to its recognition by the acetyl LDL receptor. Biochimica et Biophysica Acta 917: 337-50; 1987.

19. Lentz SR, Sadler JE. Inhibition of thrombomodulin surface expression and protein C activation by the thrombogenic agent homocysteine. Journal of Clinical Investigation 88: 1906-14; 1991.

20. Nygard O, Vollset SE, Refsum H, et al. Total plasma homocysteine and cardiovascular risk profile. JAMA 274(19): 1526-33; 1995.

21. Selhub J, Jacques PF, Wilson PWF, et al. Vitamin status and intake as primary determinants of homocysteinemia in an elderly population. JAMA 270: 2693-8; 1993.

22. Glueck CJ, Shaw P, Lang JE, Tracy T, Sieve Smith L, Wang Y. Evidence that homocysteine is an independent risk factor for atherosclerosis in hyperlipidemic patients. American Journal of Cardiology 75 : 132-6; 1995.

23. Selhub J, Jacques PF, Bostom AG, et al. Association between plasma homocysteine concentrations and extracranial carotid artery stenosis. New England Journal of Medicine 332: 286-91; 1995.

24. den Heijer M, Blom HJ, Gerrits WBJ, et al. Is hyperhomocysteinemia a risk factor for recurrent venous 
thrombosis? Lancet 345: 882-5; 1995.

25. Landgren F, Israelsson B, Lindgren A, et al. Plasma homocysteine in acute myocardial infarction: homocysteine lowering effect of folic acid. Journal of Internal Medicine 237: 381-8; 1995.

26. Perry IJ, Refsum H, Morris RW, et al. Prospective study of serum total homocysteine concentration and risk of stroke in middle aged British men. Lancet 346: 1395-8; 1995.

27. Naureth HJ, Joosten E, Riezler R, et al. Effects of vitamin B12, folate, and vitamin B6 supplements in elderly people with normal serum vitamin concentrations. Lancet 346: 85-9; 1995.

28. van den Berg M, Franken DG, Boers GHJ, et al. Combined vitamin B6 plus folic acid therapy in young patients with atherosclerosis and hyperhomocysteinemia. Journal of Vascular Surgery 20: 933-40; 1994.

29. Wilcken DEL, Wilcken B, Dudman NPB, et al. Homocystinuria-the effects of betaine in the treatment of patients not responsive to pyridoxine. New England Journal of Medicine 309: 448-53; 1983.

30. Andersson A, Brattstrom L, Israelsson B, et al. Plasma homocysteine before and after methionine loading with regard to age, gender, and menopausal status. European Journal of Clinical Investigation 22: 79-87; 1992.

31. Brattstrom L, Lindgren A, Israelsson B, et al. Homocysteine and cysteine determinants of plasma levels in middle aged and elderly subjects. Journal of Internal Medicine 236: 633-41; 1994.

32. Selhub J, Jacques PF, Wilson PWF, et al. Vitamin status and intake as primary determinants of homocysteinemia in the elderly. JAMA 270: 2693-8; 1994.

33. Morrison HI, Schaubel D, Desmeules M, et al. Serum folate and risk of fatal coronary heart disease. JAMA 275: 1893-6; 1996.

34. Lindenbaum J, Rosenberg IH, Wilson PWF, et al. Prevalence of cobalamin deficiency in the Framingham elderly population. American Journal of Clinical Nutrition 60: 2-11; 1994.

35. Brattstrom L, Israelsson B, Jeppsson JO, et al. Folic acid an innocuous means of reducing plasma homocysteine. Scandanavian Journal of Clinical and Laboratory Investigation 48: 2150-21; 1988.

36. Allen Rh, Stabler SP, Savage DG, et al. Diagnosis of cobalamin deficiency. I: Usefulness of serum methylmalonic acid and total homocysteine concentrations. American Journal of Hematology 34: 90-8; 1990.

37. Oakley GP, Adams MJ, Dickinson CM. More folic acid for everyone, now. Journal of Nutrition 126: 751S-5S; 1996.

38. Savage DG, Lindenbaum J. Folate cobalamin interactions. In: Bailey LB, editor. Folate in Health and Disease. New York, NY: Marcel Dekker Inc; 1995.

\section{BIOGRAPHY}

Kelly Marie Elian is currently a fourth-year dietetic student at McGill University, MacDonald campus (St. Anne de Bellevue, Quebec, Canada). Her review of the literature on the relationship between plasma homocysteine and vascular disease was conducted during her third year of dietetic study. Following receipt of 
her Bachelor of Science degree in Nutritional Science, she plans to pursue a Master's Degree at McGill University focusing on the relationship between nutrition and pregnancy.

Copyright (C) 1996 by MJM 